\title{
Postnatal sensitivity of the peripheral chemoreceptors in newborn infants
}

\author{
T HERTZBERG,${ }^{*}$ AND H LAGERCRANTZ \\ ${ }^{*}$ The Nobel Institute for Neurophysiology, Karolinska Institute, and Department of Pediatrics, Karolinska \\ Hospital, Stockholm, Sweden
}

SUMmARY The peripheral chemoreflex was tested in healthy term infants by measuring the ventilatory response to $100 \%$ oxygen over 30 seconds. Minute ventilation did not change when studied two to six hours after birth. By contrast, at 2-6 days of age a mean decrease of $9 \cdot 8 \%$ was noted, the difference between the groups being highly significant. There were no significant changes in respiratory rate. It is concluded that the chemoreflex is less active immediately after birth than it is a few days later, possibly due to a resetting of the sensitivity of the peripheral chemoreceptors from the fetal state, with its relatively low arterial oxygen tension to the higher postnatal concentrations.

Carotid chemoreceptors have been reported to be silent in fetal sheep and it has been assumed that they are activated at or shortly after birth by the sympathetic nervous system. ${ }^{1}$ Studies in human neonates have shown active chemoreflexes both in term and preterm infants. ${ }^{23}$ Girard et al, on the other hand, found no effect on ventilation when they produced 'physiological chemodenervation' with $100 \%$ oxygen in infants 1-19 hours of age, and speculated that peripheral chemoreceptors were not activated until some time after birth. ${ }^{4}$

The concept that the carotid chemoreceptors do not respond before birth has recently been challenged by Blanco et al, ${ }^{5}$ who found that peripheral chemoreceptors in fetal sheep were active and responsive. ${ }^{5}$ They could not, however, show any spontaneous chemoreceptor activity in newborn lambs until a few days after birth. Their interpretation was that the 'set point' of the sensitivity of the peripheral chemoreceptors changed from the low oxygen tension in the fetus to the adult range during the first few days of life.

These contradictory findings prompted us to reinvestigate peripheral chemoreceptor activity in newborn infants.

\section{Subjects and methods}

Sixteen newborn infants born at term ( 12 boys and four girls) were studied. They were all delivered vaginally after uncomplicated pregnancies. In the group of the 11 mothers whose infants were studied on the day of birth six received pudendal nerve blocks and six were given pethidine during labour; four received both types of analgesia. There were no signs of asphyxia, all Apgar scores being 7 or more at one minute. Mean birth weight was $3815 \mathrm{~g}$, ranging $2910-4530$. All infants were healthy on examination before the test. The parents were approached in the delivery room and informed consent was obtained. The study was approved by the local ethical committee.

To determine the influence of the peripheral chemoreceptors on normal breathing in air, we assessed exhaled volumes before and after administration of pure oxygen. Volume measurements were made with a Greer Electrospirometer CS5 (Mercury Electronics, Scotland), the flowhead being connected by a short tube to an infant face mask (Bennett Medical Equipment, Los Angeles, California, USA). The flowhead was connected distally via a $T$ tube to a warmed humidified stream of air set at five $1 /$ minute, which could be rapidly changed to pure humidified oxygen.

Expiratory flow was integrated electronically to obtain tidal volumes. The total dead space of the system was estimated to be $7 \mathrm{ml}$. We had no access to a capnograph at the time of the present study, so we assessed the build up of carbon dioxide in the mask in a separate experiment using the same equipment. Inspired carbon dioxide was measured with a capnograph during steady state conditions and was less than $1 \%$. The system was calibrated with 10,15 , and $20 \mathrm{ml}$ of humidified air and oxygen. 
Volume readings were about $10 \%$ higher with pure oxygen than with air due to the difference in the viscosity of the gases. Because exhaled rather than inhaled volumes were measured and the oxygen concentrations of the individual breaths change during the breathing cycle, no corrections to allow for this difference were made. In some cases inspired oxygen concentrations were estimated indirectly using a nitrogen analyser (505 Nitralyzer, MedScience, Needham Heights, Massachusetts, USA) and taking samples from the tubing between the mask and the flowhead. Tidal flow, tidal volumes, and nitrogen concentrations were recorded on a chart recorder (Mingograph, ElemaSchönander, Sweden).

The test was performed during quiet sleep two to six hours after birth and at 2-6 days of age. Quiet sleep was assessed by behavioural criteria, including regular breathing apart from sighs and the absence of eye movements and gross body movements apart from startles.

The mask was gently applied to the infant's face and then firmly held; most babies tolerated this well. In some cases they became restless but soon returned to quiet sleep. After the readings had become stable, recordings were made for at least 30 seconds before air was substituted for the oxygen for the same length of time. In those cases where the test was repeated in the same session at least three minutes elapsed between the runs.

Tidal volumes and respiratory rate were calculated during a 30 second control period and 30 seconds of oxygen administration. Percentage changes in exhaled volumes and changes in breathing frequencies were determined using each infant as his or her own control. Differences in respiratory rate and percentage changes in ventilation were analysed by

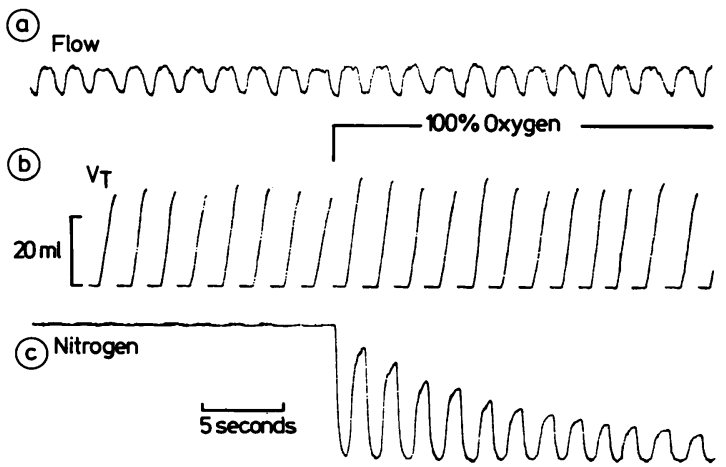

Fig 1 Tracing from hyperoxic test in a 6 hour old infant. From top to bottom: flow, integrated tidal volumes $\left(V_{T}\right)$, and expired nitrogen.
Student's $t$ test for independent samples, regarding each test as an independent event. All values are expressed as mean (SD).

\section{Results}

The test performed two to six hours after birth comprised 13 trials in 11 infants. At 2-6 days of age we carried out 12 tests in eight babies. Sequential recordings - that is, acceptable tests both on the day of birth and a few days later-were obtained in three infants. A representative recording is shown in fig 1.

During the control period ventilation was $211(80)$ $\mathrm{ml} / \mathrm{kg} /$ minute in the younger group and 218 (56) $\mathrm{ml} / \mathrm{kg} /$ minute among the older babies. There was no correlation between ventilation during the control period and the response to hyperoxia. Results were reproducible in half the infants when the test was

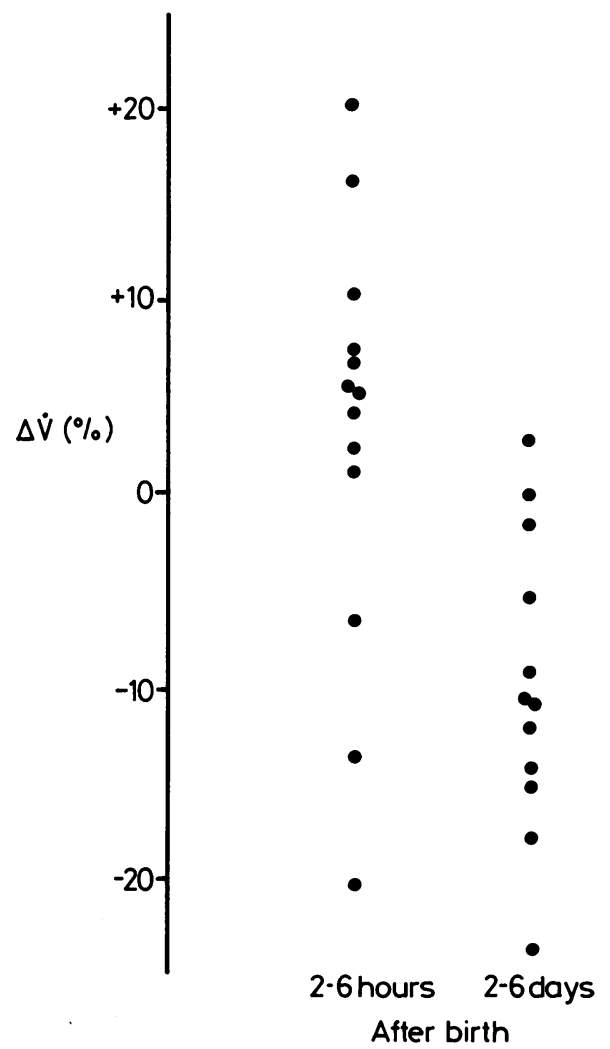

Fig 2 Percentage change in exhaled volumes $(\triangle \dot{V} \%)$ on exposure to $100 \%$ oxygen for infants 2-6 hours after birth (left) and at 2-6 days of age (right). 
repeated at the same age. The most the results differed between tests was $13 \cdot 6 \%$.

The percentage change in ventilation when the infants were made hyperoxic is shown in fig 2 . There was an insignificant mean increase of $3 \cdot 1(11 \cdot 1) \%$ in the youngest group. In contrast, the older infants decreased their minute ventilation by $9.8(7.7) \%$ $(\mathrm{p}<0.01)$. The difference in ventilatory response between the two age groups was significant $(p<0 \cdot 01)$. Among the infants $2-6$ hours old respiratory rate changed from $40.0 \quad(12.0) /$ minute to $38 \cdot 8(10 \cdot 3) /$ minute. In the infants $2-6$ days old the mean frequency decreased from 38.4 (8.9)/minute to $36.6(6 \cdot 8) /$ minute. The changes in respiratory rate were not significant. The reduction in ventilation was therefore mainly due to a decrease in tidal volume.

\section{Discussion}

Prolonged exposure to hyperoxia causes an initial decrease in ventilation followed by a sustained increase. The primary response is ascribed to the peripheral chemoreceptors; the late increase may be due to several factors-for example, changes in cerebral blood flow and arterial carbon dioxide tension $\left(\mathrm{PCO}_{2}\right){ }^{6}$

The ' $\mathrm{O}_{2}$ test' was proposed by Dejours ${ }^{7}$ to estimate the influence of the peripheral chemoreceptors on ventilation. A short term stimulus affects the arterial chemoreceptors rapidly, and the effect may be recorded before any secondary influences are apparent. The method was first used in newborn infants by Girard et al to evaluate the postnatal activity of the peripheral chemoreflex. ${ }^{4}$ Purves $^{x}$ criticised the test, pointing out that a single breath of oxygen may not raise arterial oxygen tension $\left(\mathrm{PO}_{2}\right)$ sufficiently because of the high degree of venous admixture in arterial blood caused by shunts in the newborn. To circumvent this problem we used a somewhat longer oxygen exposure. We believe that 30 seconds is long enough to raise arterial $\mathrm{PO}_{2}$ to sufficient hyperoxic concentrations without being affected by the normal fluctuations in breathing volumes. The time should still be short enough to avoid central chemoreceptor stimulation due to consequent increases in $\mathrm{PCO}_{2}$.

A feature of this study was the strict age grouping in the immediate postnatal period. The finding that hyperoxia decreases ventilation in infants $2-6$ days old while minute ventilation is less affected two to six hours after birth suggests an increasing influence of the hypoxic ventilatory drive during the first days of life. Our results are consistent with the finding of a resetting of the sensitivity of the peripheral chemoreceptors after birth in newborn lambs.
Jansen et al showed that the peripheral chemoreceptors are not essential for intrauterine breathing movements or for the establishment of effective breathing at birth."

Our figures for the decrease in ventilation when the older infants were made hyperoxic are somewhat lower than those presented by others. This may be explained by the fact that we integrated the expired flows. Pure humidified oxygen gave about $10 \%$ higher readings than air on the pneumotachograph. We did not correct for this because the composition of the expired gas changes breath by breath. Because the differences between the groups were based on percentage changes in ventilation before and after hyperoxia, such a correction would most likely not have influenced the results.

There may be some doubt about assessing the relative contribution of the hypoxic drive to the central controller of ventilation from the ventilatory output, which may in turn be affected by several other factors. These include auditory, tactile, and thermal stimuli as well as the analgesics given to the mother during labour, and they are probably not of constant and equal importance in all babies. This may in part explain the variation in responses, both between different runs in the same infant and between different infants. Thus the test results of individual babies are difficult to interpret. The influence of a possible build up of carbon dioxide in the mask cannot be excluded. No -correlation was found, however, between ventilation during the control period and the hyperoxic response. We found no obvious explanation for the finding that some of the older infants did not decrease ventilation when made hyperoxic. There may be variation between babies in the time needed for the postnatal adaptation. The overall picture is, however, clear: there is a difference in response between the age groups, and the three infants tested at different postnatal ages all showed an increased response over the first days of life.

Several investigators have found an active chemoreflex in newborn infants, although the age of their subjects has not been confined to the first days of life. Cross and Warner reported a decrease in ventilation in response to oxygen even during the first days of life. ${ }^{2}$ Interestingly, their babies studied on more than one occasion showed more pronounced decreases as they grew older, a finding which is not commented on by the authors but is in agreement with our results. Girard et al found that a single breath of $100 \%$ oxygen failed to affect ventilation in infants 1-19 hours old while older babies responded with a decrease in ventilation. ${ }^{4}$

The postnatal arterial oxygen tension seems to influence the degree of sensitivity. People born at 
high altitude in the Andes have a weaker hypoxic response than those born at sea level. This lower sensitivity seems to be irreversible, as those born at high altitude moving to sea level and lowlanders moving to high altitude exhibit the same responses as controls remaining at high altitude and sea level, respectively. ${ }^{1011}$ The same authors suggested that infants with chronic hypoxia and cyanotic heart failure from birth acquire a permanent low chemoreceptor sensitivity. ${ }^{12}$ This has been disputed by Edelman et al, however, who, using a different method, reported that patients with tetralogy of Fallot restore their sensitivity to hypoxia after corrective surgery. ${ }^{13}$

The present findings suggest, in agreement with Blanco $e t a l$, that the sensitivity of the peripheral chemoreceptors noticeably changes during the first days after birth. ${ }^{5}$ Postnatal oxygen tension seems to determine the sensitivity threshold but other perinatal factors may play a part-for example, drugs given to the mother and catecholamine concentrations in the newborn. The clinical implications may be, as pointed out by Hanson, ${ }^{14}$ that an asphyxiated infant develops a delayed resetting of the oxygen sensitivity due to prolonged hypoxemia and an increased susceptibility to hypoxemia due to low sensitivity of the peripheral chemoreceptors; this in turn leads to a further delayed resetting. This concept emphasises the importance of adequate postnatal $\mathrm{PO}_{2}$ concentrations to ensure an optimal adaptation to extrauterine life.

This study was supported by the Swedish Medical Research Council, grant No 5234, Stiftelsen Majblomman and Stiftelsen Solstickan.

\section{References}

' Purves MJ. Chemoreceptors and their reflexes with special reference to the fetus and newborn. J Dev Phyisol 1981;3:21-57.
2 Cross KW, Warner P. The effect of inhalation of high and low oxygen concentrations on the respiration of the newborn infant. J Physiol 1951;114:283-95.

${ }^{3}$ Cross KW, Oppé TE. The effect of inhalation of high and low concentrations of oxygen on the respiration of the premature infant. J Physiol 1952:117:38-55.

${ }^{4}$ Girard F, Lacaisse A, Dejours P. Le stimulus $\mathrm{O}_{2}$ ventilatoire a la période néonatale chez l'homme. J Physiol (Paris) 1960):52: 108-9.

5 Blanco CE, Dawes GS, Hanson MA, McCooke HB. The response to hypoxia of arterial chemoreceptors in fetal sheep and newborn lambs. J Physiol 1984:351:25-37.

' Cunningham DJC, Robbins PA, Wolff CB. Integration of respiratory responses to changes in alveolar partial pressures of $\mathrm{CO}_{2}$ and $\mathrm{O}_{2}$ and in arterial pH. In: Fishman AP. Cherniack NS, Widdicombe JG, eds. Handbook of physiology: the respiratory system. Bethesda: American Physiological Society, 1986.

7 Dejours P. Chemoreflexes in breathing. Physiol Rev 1962;42: 335-58.

${ }^{8}$ Purves MJ. The effect of a single breath of oxygen on respiration in the newborn lamb. Respir Physiol 1966;1:297-307.

9 Jansen AH, loffe S, Russel BJ, Chernick V. Effect of carotid chemoreceptor denervation on breathing in utero and after birth. J Appl Physiol 1981:51:630-3.

${ }^{10}$ Sørensen SC, Severinghaus JW. Respiratory sensitivity to acute hypoxia in man born at sea living at high altitude. $J$ Appl Physiol 1968:25:211-6.

1 Sørensen SC, Severinghaus JW. Irreversible respiratory insensitivity to acute hypoxia in man born at high altitude. J Appl Physiol 1968;25:217-20.

12 Sørensen SC, Severinghaus JW. Respiratory insensibility to acute hypoxia persisting after correction of tetralogy of Fallot. J Appl Physiol 1968:25:221-3.

13 Edelman NH, Lahiri S, Braudo L, Cherniack NS, Fishman AP. The blunted ventilatory response to hypoxia in cyanotic congenital heart disease. $N$ Engl J Med 1970;282:405-11.

14 Hanson MA. Maturation of the peripheral chemoreceptor and CNS components of respiratory control in perinatal life. In: von Euler C, Lagercrantz H, eds. Neurobiology of the control of breathing. New York: Raven Press, 1987.

Correspondence to $\mathrm{Dr} \mathrm{T}$ Hertzberg, The Nobel Institute for Neurophysiology, Karolinska Institute, Box 60400), S-104 01 Stockholm, Sweden.

Reccived 18 May 1987 